\title{
A spiral phantom for IMRT and tomotherapy treatment delivery verification
}

\author{
Bhudatt Paliwal| ${ }^{\mathrm{a})}$ and Wolfgang Tomé \\ Departments of Human Oncology and Medical Physics, Medical School, University of Wisconsin, \\ Madison, Wisconsin 53792 \\ Susan Richardson \\ Department of Human Oncology, Medical School, University of Wisconsin, Madison, Wisconsin 53792 \\ T. Rockwell Mackie \\ Departments of Human Oncology and Medical Physics, Medical School, University of Wisconsin, \\ Madison, Wisconsin 53792
}

(Received 11 January 2000; accepted for publication 28 August 2000)

\begin{abstract}
A solid water/cylindrical phantom is machined to create a spiral cavity for placing radiographic or radiochromatic film in a spiral configuration. This spiral phantom is used to sample, predict, and measure data in three-dimension subspace. The predicted data are obtained by projecting the patient plan data on the spiral phantom in the treatment planning software. The measured data are obtained by irradiating the spiral phantom (with film in the spiral cavity) as per the treatment plan. The predicted and measured data are converted to a two-dimensional matrix and plotted as a spiralogram. Comparison of these predicted and measured spiralograms provides a quantitative comparison and thus validation of treatment delivered as planned. The spiral phantom is a simple, costeffective approach to sample 3D data from complexly shaped, intensity modulated or compensated multiple beams. A software script is being written to automate the entire process of projection, data sampling, and comparison. Design aspects and some examples of dose verification are presented. The usefulness of the spiral phantom for intensity modulated radiation therapy and dynamic field shaping are discussed. (C) 2000 American Association of Physicists in Medicine.
\end{abstract}

[S0094-2405(00)01911-8]

Key words: IMRT treatment plan verification, QA phantom

\section{INTRODUCTION}

The advancements in the planning and delivery of curative radiation therapy are likely to utilize complex intensity modulated radiation therapy (IMRT) protocols. This process may involve pulse-by-pulse change in intensity, use of multileaf collimators, and dynamic wedges, or combinations of all of the modifiers, as proposed in tomotherapy. Because of these complexities, it is important to develop radiation dose delivery verification and quality assurance process specifically addressing these tools. We discuss in this paper a phantom design that allows the user to qualitatively compare a predicted dose distribution to the actual delivered one by qualitatively comparing the planned unique dose signature imprinted on a two-dimensional (2D) subspace that has the form of a spiral to a measured one.

Over many decades a large number of geometric and anthropomorphic phantoms have been developed to verify the likely radiation dose delivered to the patient for a planned treatment. Most often the choice of detectors placed in the phantoms has been ionization chambers, diodes, and radiographic film (cf. Refs. 1-11 and references therein). More recently dichromic films, phosphorescent screens, Frickeinfused agarose gel type, and PAG gel type detectors also have been used (cf. Refs. 1, 12-16). It has been found that the limit of spatial resolution of gel-type detectors is due to the MR voxel size (cf. Ref. 12), which for the spiral phantom would be $2.34 \mathrm{~mm}^{3}$, this assumes a MRI slice thickness of
$1.5 \mathrm{~mm}$ and a pixel size of $1.25 \mathrm{~mm}$. Hence, using gel-type detectors the spatial resolution would be limited to 2.34 $\mathrm{mm}^{3}$. Furthermore, one has to take into account the high cost and time required for MRI scanning, as well as the poor signal to noise ratio in measured dose distributions in reading out gel-measured dose distributions (cf. Ref. 13). Therefore, film/screen systems continue to be most practical and cost effective, and also as pointed out previously provide better spatial resolution compared to Fricke and PAG gels, diodes, and ionization chambers.

The conventional use of the film/screen type has always been in a configuration in which square blocks are used and multiple films are stacked either parallel or perpendicular to the beam. In our design we propose a cylindrical phantom with a spiral cavity to place the screen/film system. The advantages of this configuration are that the radiation beam is always perpendicular to the $\mathrm{film} / \mathrm{screen}$ system, the beams always intersect the cavity often at several depths, and one obtains a sampling of the three-dimensional dose space using a two-dimensional dose subspace that extends through the whole phantom. The details of the spiral system are given in Fig. 1.

\section{MATERIALS AND METHODS}

A solid water cylindrical phantom of $30 \mathrm{~cm}$ diameter and $15 \mathrm{~cm}$ height was machined to create an archimedian spiral cavity for placing either radiographic or radiochromic film. 


\section{Spiral Phantom}
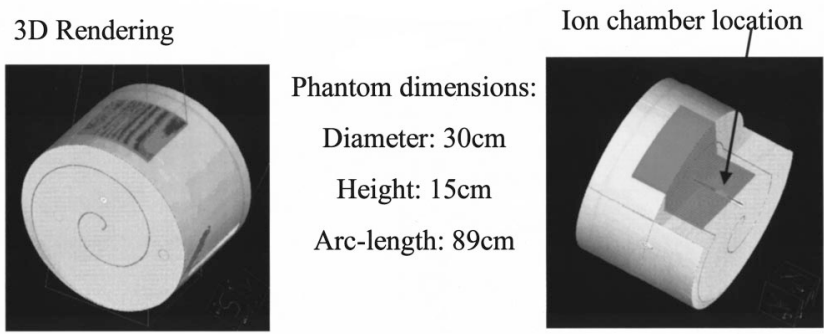

FIG. 1. The image on the left-hand side shows a 3D holographic rendering of the phantom with the surface projection of a treatment beam placed on the center of the phantom. The image on the right-hand side shows a crosssection view that exposes the location of the ion chamber.

The arclength of the spiral cavity in the transverse plane is $89 \mathrm{~cm}$. The phantom was also machined in several locations to place an ionization chamber to verify the delivered dose at these points using a calibrated ionometric system. Figure 1 is a rendered image of the phantom. It also shows a cut plane to indicate the location of an ionization chamber.

Kodak XV film is placed in the spiral cavity and the phantom is placed in a light tight enclosure consisting of a cylinder of previously exposed film. To obtain a film that has a length of $86.36 \mathrm{~cm}$ an $11 \times 17$ film is cut in half to yield two $5.5 \times 17$ pieces, these pieces are then taped at the sides at the joining ends to avoid slippage in the phantom. Straps are placed around the circumference of the phantom and tightened; this constricts the spiral and helps to eliminate air gaps as far as possible. However, there is a slight air gap at the outermost region of the machined spiral, which may account for the discrepancy between the measured and predicted relative doses in that region. The straps are placed such that they do not interfere with the experiment (Fig. 2).

After irradiation, the films were processed using a Kodak RP X-OMAT processor. All films came from the same film batch and were processed under the same conditions. Since we are interested in relative rather than absolute dosimetry, the change in processor conditions from the time the calibration curve was created to when the spiral phantom films were developed is not considered.

A calibration curve was obtained using a step wedge supplied by RIT113 software (Radiological Imaging Technology, Colorado Springs, CO). The film was scanned using an Argus II scanner (Agfa, Mortsel, Belgium), and the dose for each step was entered, producing an optical density to dose conversion table. Films from the spiral phantom were subsequently scanned in a similar manner and the doses were found which were then converted to relative doses by normalizing to the global maximum.

As pointed out previously the three-dimensional dose subspace, which samples the entire dose space is represented by an archimedean spiral. An archimedian spiral is the trajectory of a point that moves with constant velocity $v$ along a rod, which rotates at constant angular velocity $\omega$ about one of its end points. This trajectory can be written in terms of polar coordinates as follows:

$$
\rho=a|\varphi|,
$$

where

$$
a=v / \omega>0, \quad-\infty<\varphi<\infty .
$$

\section{Correlation of Data}

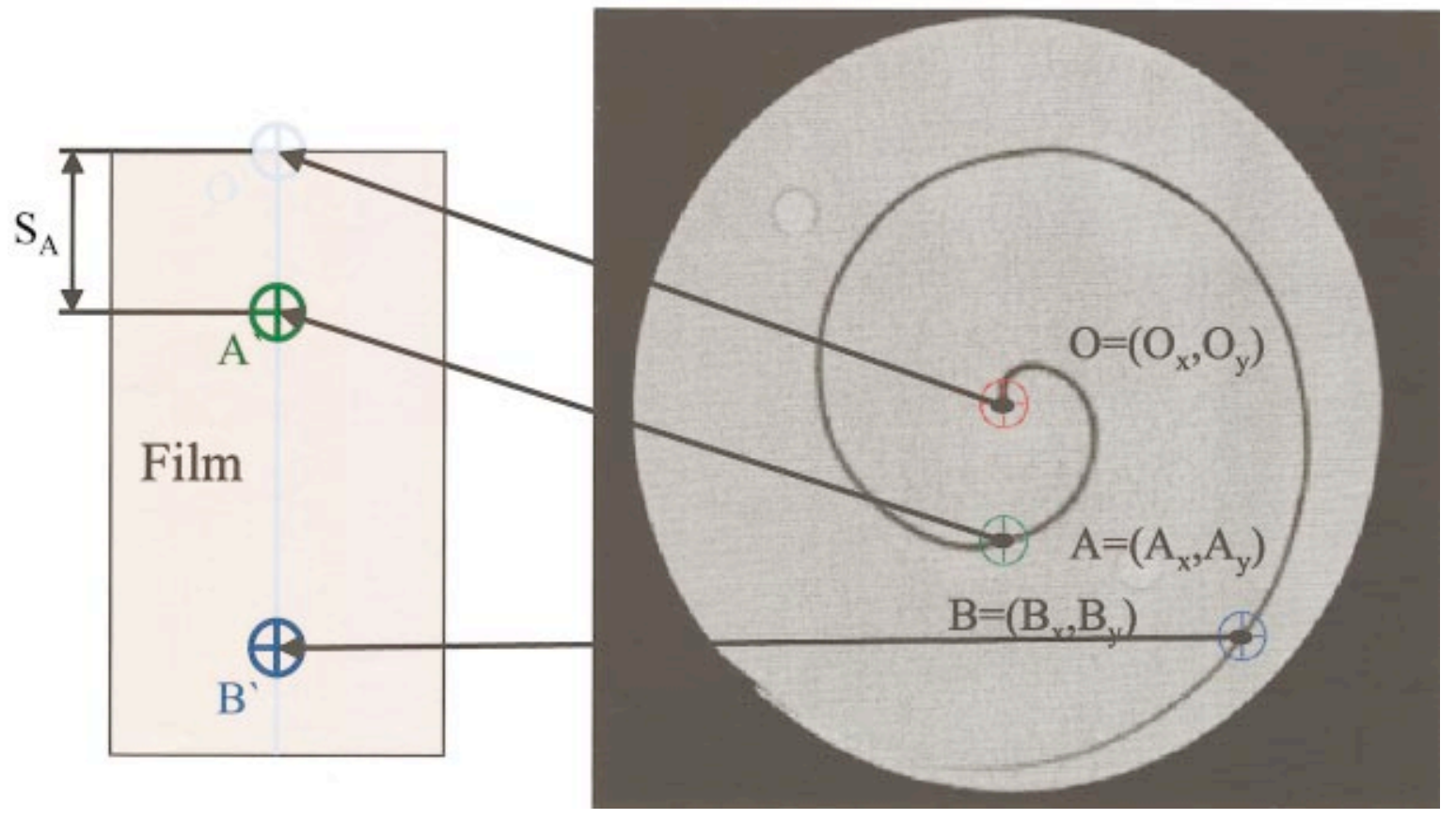

FIG. 2. Correlation of the film and the phantom space data for the central axis of the film. The transformation from general affine coordinates to spiral arclength, which uniquely relates points in the three-dimensional dose subspace to the corresponding points on the film, is illustrated. 


\section{Projection of Patient Plan on the Spiral phantom}

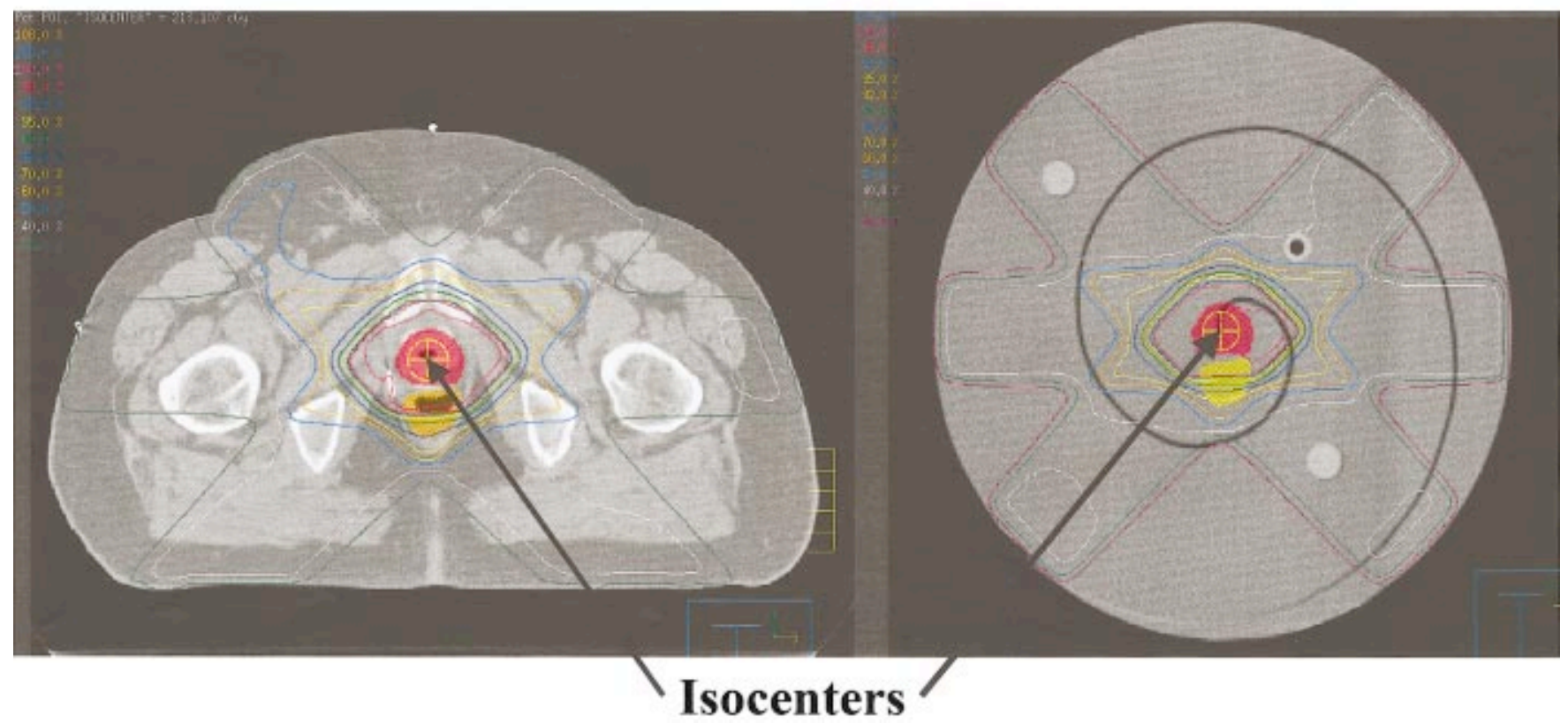

FIG. 3. The image on the left-hand side shows the dose distribution obtained in a typical six field prostate beam setup, while the image on the right-hand side shows the isodose distribution obtained after the plan has been projected onto the spiral phantom which includes corrections for the change in the contour of the patient to that of the cylindrical configuration of the phantom.

We have selected for our work the branch of the spiral with $0 \leqslant \varphi<\infty$. In terms of affine coordinates, the Archimedean spiral can be given the following parametrization:

$$
x(\varphi)=a \varphi \cos (\varphi), \quad y(\varphi)=a \varphi \sin (\varphi),
$$

where

$$
0 \leqslant \varphi<\infty .
$$

As can be seen from Fig. 3, one can uniquely relate each point in the three-dimensional dose subspace to a point along the film. In fact two of three coordinates, namely $x$ and $y$, are collapsed into a single coordinate - that of arclength. Hence, we achieve a dimensional reduction that allows us to qualitatively and quantitatively compare predicted and actual dose distributions in the three-dimensional subspace by comparing two-dimensional matrices. This transformation is straightforwardly obtained by evaluating the arclength along the spiral as illustrated in Fig. 3 in terms of affine coordinates:

$$
s(\varphi)=\int_{0}^{\varphi} \sqrt{\dot{x}^{2}(t)+\dot{y}^{2}(t)} d t=a \int_{0}^{\varphi} \sqrt{1+t^{2}} d t .
$$

This integral can be straightforwardly evaluated to yield in the terminology of Fig. 3:

$$
S_{A}=\frac{1}{2 a} \sqrt{x^{2}+y^{2}} \sqrt{a^{2}+x^{2}+y^{2}}+\frac{a}{2} \sinh ^{-1}\left(\frac{\sqrt{x^{2}+y^{2}}}{a}\right),
$$

where

$$
x=A_{x}-O_{x}, \quad y=A_{y}-O_{y} .
$$

Using this expression one can generate the predicted spiralogram for a particular plan under consideration by projecting the previously developed treatment plan using the patient anatomy onto the spiral phantom. In effect we replace the patient with the spiral phantom without altering the weighting or intensity patterns obtained previously using inverse planning. However, the projected dose calculation includes corrections for change in the contour of the patient to that of the cylindrical configuration of the spiral phantom. As a particular example a six beam setup with the planned dose distribution in the patient and the corresponding projected dose distribution in the phantom are shown in Fig. 3.

\section{RESULTS}

The measured data are obtained by irradiating the spiral phantom with the film placed in the spiral cavity. In this experiment no use was made of the ion chamber cavities and the cavities were closed using solid water plugs that had the exact chamber dimensions. However, if desired the user can obtain readings at two off-axis points which can then be compared to the predicted doses at these points. All the machine settings are determined from the treatment plan. The film is then scanned as described previously, and the measured data for the central axis plane are converted to a $2 \mathrm{D}$ 


\section{Measured and Predicted Spiralograms}

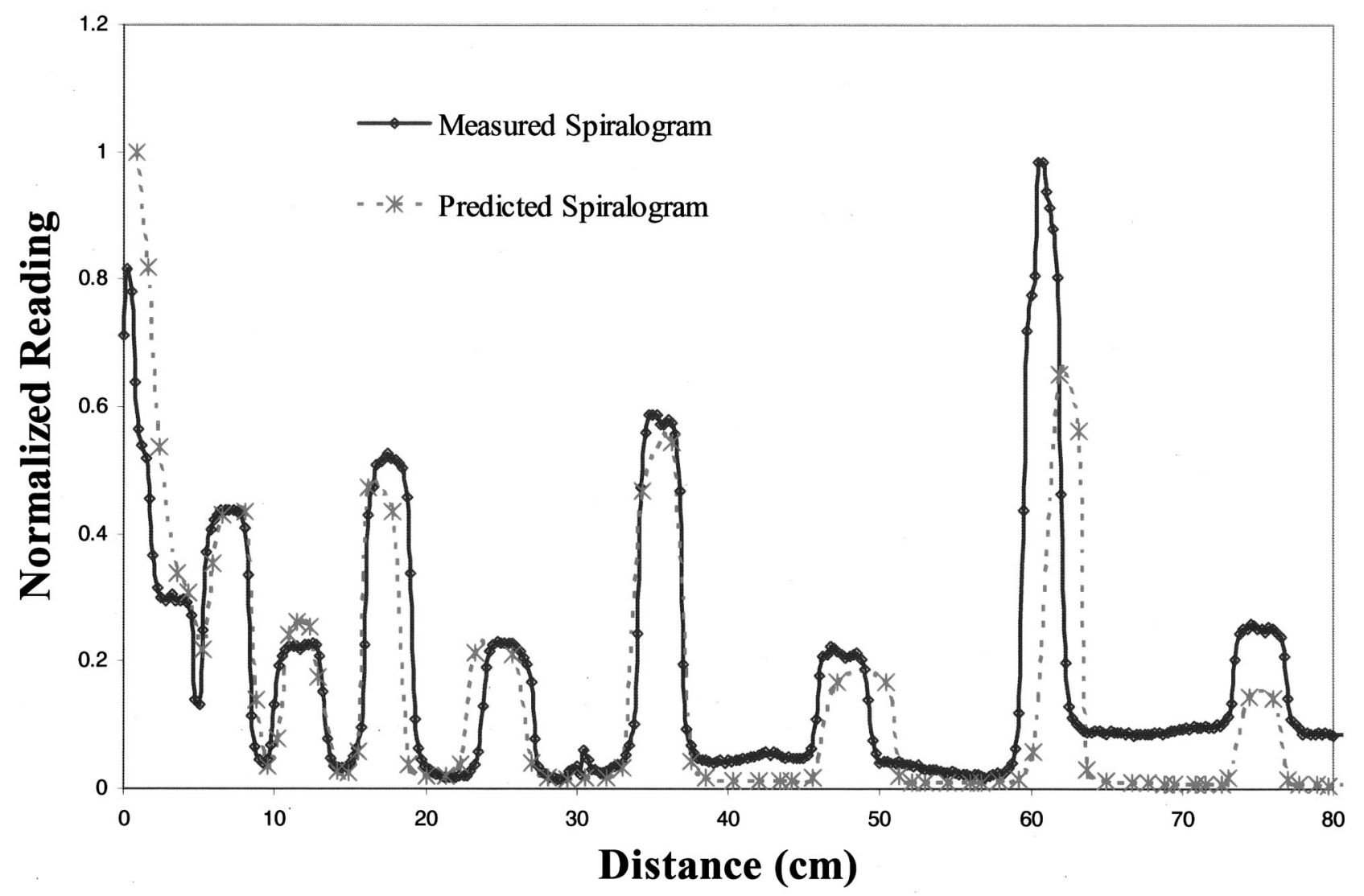

FIG. 4. The predicted and actually measured Spiralogram using XV film is shown. Each of the two data sets has been normalized to its global maximum, respectively. The data have not been extended beyond $70 \mathrm{~cm}$ of arclength since the remaining part of the spiral lies in the buildup region in which the prediction of dose from the treatment planning system is questionable.

matrix and plotted as a spiralogram after the data has been normalized to its global maximum. An overlay of predicted and measured spiralogram for three, $6 \mathrm{MV}$, isocentric beams, $3 \times 3 \mathrm{~cm}$ fields is shown in Fig. 4. The data beyond $70 \mathrm{~cm}$ of arclength is questionable, since the remaining part of the spiral lies in the buildup region in which the prediction of dose from the treatment planning system is doubtful. It has been pointed out in the literature (cf. Refs. 17 and 18) that the calibration conditions are important since for radiographic film the sensitomtric curve can vary with both field size and depth in the phantom due to increases in lateral photon phantom scatter. Therefore, in general the calibration curves used should be both depth- and field-size specific. ${ }^{19}$ However, it has been recently pointed out [cf. Ref. 20, Figs. 2(a), 2(b), and 4] that the net optical density changes with depth and circular field diameter negligibly for doses up to $1.4 \mathrm{~Gy}$ and for a $20 \times 20$ field for doses up to $0.6 \mathrm{~Gy}$ and for doses less than $0.8 \mathrm{~Gy}$ the error is less than $10 \%$. Since our phantom is to be used in the realm of helical tomotherapy and small field IMRT it is reasonable to use a single calibration curve and to neglect both depth and field size corrections as long as the conditions outlined previously are met.

\section{DISCUSSION}

Comparison of the predicted and measured data provides a visually qualitative assessment of the treatment to be delivered. As can be seen from Fig. 4 the predicted and measured Spiralogram are qualitatively in good agreement for the first $70 \mathrm{~cm}$ of arclength, i.e., in the central part of the spiral phantom. The agreement is not as good from $70 \mathrm{~cm}$ of arclength to $86.36 \mathrm{~cm}$, i.e., at the rim of spiral phantom. On the one hand, the part of spiral with an arclength of larger than $70 \mathrm{~cm}$ lies in the buildup region and as pointed out previously the doses predicted by the treatment planning system in this region are questionable. While on the other hand, due to the nonlinearity of the film response, the dose in this region may be either over- or underestimated through film dosimetry. However, one can clearly see the value of this phantom as a qualitative assessment tool for complex isodose distributions as obtained from inverse planing algorithms. Additionally, the entire process of developing predicted and measured spiralograms can be automated by incorporating the outlined manual procedure into the treatment planning software and the film scanning RIT113 software. 


\section{CONCLUSIONS}

The spiral phantom samples, predicts, and measures data in a three-dimensional (3D) subspace, which is reduced to a single 2D matrix. The predicted and measured 2D matrices are easily plotted in terms of a spiralogram and compared.

One can think of the spiralogram as sort of depth dose curve along the spiral trajectory, or as the dose one would receive if one were to travel along the radial direction with constant velocity in an inertial system, which rotates at a constant angular velocity, $\omega$. The observed differences between the predicted and measured spiralograms are correctable by improvements in film positioning and calibration. We are in the process of redesigning the film loading mechanism. The new design consists of $1.5-\mathrm{cm}$-diam solid water rod that has a machined out groove into which the film is inserted and then pinned into place. Together with the film, this rod is then inserted into the phantom. The pin marks provide a unique way of determining the origin of the film once it has been exposed. The predicted and measured spiralograms shown in Fig. 4 clearly demonstrate the feasibility of using the spiral phantom for pretreatment evaluation of a treatment plan. In our opinion the power of this method lies in the fact that it provides a quick and easy qualitative way of verifying a treatment plan through visual comparison of a predicted and measured Spiralogram. The process of generating the predicted spiralogram, film scanning, and comparative analysis can be automated very easily. Future work includes development of automated scripts and comparison of dose distributions for intensity modulated complex and conformal treatment deliveries.

a) Author to whom correspondence should be addressed; electronic mail: bpaliwal@facstaff.wisc.edu

${ }^{1}$ M. J. Butson, P. K. N. Yu, and P. E. Metcalfe, "Extrapolated surface dose measurements with radiochromic film,' Med. Phys. 26, 485-488 (1999).

${ }^{2}$ B. A. Fraass and D. L. McShan, “3-D treatment planning. I. Overview of a clinical planning system," in The Use of Computers in Radiation Therapy, edited by I. A. D. Bruinvis, P. H. van der Giessen, H. J. van Kleffens, and F. W. Wittkamper (Elsevier/North-Holland, New York, 1987), pp. 273-276.

${ }^{3}$ B. A. Fraass, "Clinical application of 3-D treatment planning," in Advances in Radiation Oncology Physics, Dosimetry, Treatment Planning and Brachytherapy, edited by J. A. Purdy (AIP, Woodbury, NY, 1992), pp. 967-997.

${ }^{4}$ B. A. Fraass, M. K. Martel, and D. L. McShan, "Tools for dose calculation verification and QA for conformal therapy treatment techniques,", in Proceedings of the 11th International Conference on the Use of Computers in Radiation Therapy, edited by A. R. Hounsell, J. M. Wilkinson, and P. C. Williams (Medical Physics Publishing, Madison, WI, 1994), pp. 256-257.

${ }^{5}$ E. C. McCullough and A. M. Krueger, "Performance evaluation of computerized treatment planning systems for radiotherapy: External photon beams,' Int. J. Radiat. Oncol., Biol., Phys. 6, 1599-1605 (1980).

${ }^{6}$ R. Mohan, G. Barest, L. J. Brewster, C. S. Chui, G. J. Kutcher, J. S. Laughlin, and Z. Fuks, " A comprehensive 3-dimensional radiation treatment planning system,' Int. J. Radiat. Oncol., Biol., Phys. 15, 481-495 (1988).

${ }^{7}$ D. A. Low, J. F. Dempsey, R. Venkatesan, S. Mutic, J. Markman, E. M. Haacke, and J. A. Purdy, "Evaluation of polymer gels and MRI as a 3-D dosimeter for intensity-modulated radiation therapy,' Med. Phys. 26, 1542-1551 (1999).

${ }^{8}$ J. A. Purdy, B. H. Williams, J. Michalski, and J. D. Cox, "Multiinstitutional clinical trials: 3-D conformal radiotherapy quality assurance,' Radiat. Ther. Oncol. 29, 255-263 (1996).

${ }^{9}$ U. F. Rosenow et al., "Quality assurance in treatment planning. Report from the German Task Group, in Ref. 2, pp. 45-48.

${ }^{10}$ J. Van Dyk, R. B. Barnett, J. E. Cygler, and P. C. Shragge, 'Commissioning and quality assurance of treatment planning computers,' Int. J. Radiat. Oncol., Biol., Phys. 26, 261-273 (1993).

${ }^{11}$ C. F. Westmann, B. J. Mijnheer, and H. J. van Kleffens, "Determination of the accuracy of different computer planning systems for treatment with external photon beams,', Radiother. Oncol. 1, 339-347 (1984).

${ }^{12}$ R. J. Schulz, A. F. DeGuzman, D. B. Nguyen, and J. C. Gore, “Dose response curves for Fricke-infused argose gels as obtained by nuclear magnetic resonance," Phys. Med. Biol. 35, 1611-1622 (1990).

${ }^{13}$ S. L. Meeks, F. J. Bova, M. J. Maryanski, L. A. Kendrick, M. K. Ranade, J. M. Buatti, and W. A. Friedman, "Image registration of BANG® gel dose maps for quantitative dosimetry verification,' Int. J. Radiat. Oncol., Biol., Phys. 43, 1135-1141 (1999).

${ }^{14}$ D. M. Duggan, C. W. Coffey II, J. L. Lobdell, and M. C. Schell, "Radiochromic film dosimetry of a high dose rate beta source for intravascular brachytherapy,', Med. Phys. 26, 2461-2464 (1999).

${ }^{15}$ A. R. Farajollahi, "An investigation into the applications of polymer gel dosimetry in radiotherapy,'” Med. Phys. 26, 493 (1999).

${ }^{16}$ S. J. Thomas, I. D. Wilkinson, A. K. Dixon, and P. P. Dendy, "Magnetic resonance imaging of Fricke-doped agarose gels for the visualization of radiotherapy dose distributions in a lung phantom,' Br. J. Radiol. 65, 167-169 (1992).

${ }^{17}$ S. E. Burch, K. J. Kearfott, J. H. Trueblood, W. C. Sheils, J. Inhwan Yeo, and C. K. C. Wang, "A new approach to film dosimetry for high energy photon beams: Lateral scatter filtering,', Med. Phys. 24, 775-783 (1997).

${ }^{18}$ J. F. Williamson, F. M. Kahn, and S. C. Shama, "Film dosimetry of mega voltage photon beams: A practical method of isodensity-to-isodose curve conversion.' Med. Phys. 8, 94-99 (1981).

${ }^{19}$ J. J. Hale, A. T. Kerr, and P. C. Shragge, "Calibration of film for accurate megavoltage photon dosimetry,', Medical Dosimetry 19, 43-46 (1994).

${ }^{20}$ J. L. Robar and B. G. Clark, "The use of radiographic film for linear accelerator stereotactic radiosurgical dosimetry,' Med. Phys. 26, 21442150 (1999). 